The skin seems however generally somewhat thick- among the eminent gentlemen participating in the ened. My attention was particularly attracted to the debate.

color of the skin of the face. It was markedly white It seems odd that notwithstanding our experience with a bright spot over each cheek. This is not con- in these operations has so markedly increased since stant nor is the redness of the nose, which she tells this memorable debate, there should still exist great me is sometimes present. The lips are full and high colored, the brows and lashes both of good thickness. Except when she has a nervous perspiration the skin is dry. There is frequently a puffiness under the lids although no other signs of edema. The features are neither coarse nor broad. She has never noticed any watery edema either general or local. The abdomen has felt swollen on a few occasions, but that she refers to the abdominal trouble. The thyroid gland is of small size but is distinctly apparent. I have been unable to learn of any nervous symptoms other than an irregular feeling of weakness with coldness of the hands and feet. There is no paralysis, tremor, or inco-ordination. Sight, except for some hypermetropia, is good. Hearing is normal. Smell and taste are both considerably affected by the nasal stoppage. The voice is nasal in quality. Speech is not delayed nor does there seem to be any mental heaviness or slowness.

Examination of the blood showed: red blood cor- th puscles $5,800,000$, leucocytes 4,625 , ratio 1 to 1254 , hemoglobin 65 per cent.

There have been no hemorrhages from the gums or nose. The urine showed no abnormalities. The temperature is 98.6 degrees. Appetite and digestion are fairly good. Feeling convinced from this history, that there was more than a local condition to contend with and as some of the symptoms of myxedema were present, viz.: dry thickened skin, capillary injection over the cheeks, dryness and brittleness of the nails and hair and possibly the appearance of the mucous membranes, I ordered dessicated thyroids gram 0.32 . t.i.d. She was not seen again until Dec. 4, 1896, when she reported that the powders had relieved her very much. I had ordered only enough to last ten days, but she had had no return of the symptoms until two weeks previously. The prescription was renewed. When next seen, Jan. 18, 1897, she reported having taken thirty-two powders between December 4 and January 8 . While taking them the nasal symptoms were almost entirely relieved. Examination of the nostrils shows lessening of the puffiness and waxiness. Since this date she has taken powders more or less regularly with considerable relief.

It will be seen that while the general symptoms were not absolutely confirmatory of myxedema they were marked sufficiently to induce me to try the therapentic test and in view of the happy results attending this trial I think we can safely make the diagnosis,

1423 Walnut Street.

\title{
GENERAL AND LOCAL ANESTHESIA IN
} LARYNGOLOGY AND RHINOLOGY.

Presented to the Section on Laryngology and Otology at the Forty. eighth Anuual Meeting of the American Medical Association, held at Philadelphia, Pa., June 1-4, 1897. BY JOSEPH S. GIBB, M.D.

I'rofessor of Diseases of the Throat and Nose, Philadelphia Polyclinic Surgeon-in-charge of the Throat, Nose and Ear Department, Episcopal Hospital. PHILADELPHIA, PA.

In March, $1889^{1}$, a very interesting discussion on the proper form of anesthesia to employ in nose and throat operations, took place in London. The discussion developed the widest differences of opinion

This is to be deplored. While it is of very little moment as to the particular form of anesthesia an individual specialist uses in his practice, it is of vital importance as to the teaching of the great mass of our profession by whom these operations are being performed in increasing numbers year by year. If some order could be brought out of this chaos of opinion; a few plain and safe lines to guide the busy practitioner a good work would be performed.

For convenience of study the subject will be divided into four headings, namely:

1 , Intra-nasal operations; 2 , naso-pharyngeal operations; 3 , pharyngeal operations; 4, laryngeal operations.

Intra-nasal operations. - Intra-nasal surgery has made prodigious strides since the advent of local anesthesia. Nor is this due alone to the fact that the comfort of the patient is less disturbed. By this method, we are enabled to follow each step of an operation deliberately and intelligently, and hence to do better work.

Under general anesthesia the patient is in the worst possible condition to make a satisfactory examination. The recumbent position (the only one tenable in chloroform and ether narcosis) is one unfavorable to proper illumination, an objection which has scarcely been overcome by modern electrical refinements.

The position and the anesthetic, both favor hemorrhage, which covers the field of operation and adds to the difficulties.

General anesthesia, therefore, is indicated in intranasal operations; 1 , major operations, those in which large growths are to be removed, necessitating a considerable amount of dissection, and possibly involving the external tissues; 2 , in large bony deflections of the septum, where it is necessary to break up the septum at its base; 3 , in large bony spurs in which the relative position of the outgrowth to the surrounding tissues have been accurately determined; 4 , congenital or acquired stenoses; 5 , plastic operations.

The number of cases which come under the second and third groups, namely, deflections and spurs, are small, and one finds them growing less year by year as his experience in this work grows. It is seldom we know precisely the operative procedure we will follow in an individual case prior to the operation. For instance, in pronounced cartilaginous deflections, how frequently we find after the cartilage is freed a corresponding bony deflection, with perhaps exostoses and spurs.

We are not all favored with small fingers to enable us to determine by digital examination the extent and nature of deformities, nor are all nares of sufficient dimensions to permit such examinations to those so favored.

While breaking up a deflected bony septum or the removal of bony. outgrowths are undoubtedly painful procedures, which pain no amount of local anesthesia can entirely annul, this discomfort is transient, and

I Discussion upon the paper of George stoker on anesthetics in operations on the nose and throat, British Laryngologic Association, Wednesday, March 27, 1889. 
the benefits accruing from a method which will enable us to have the field of operation under our view at all times greatly outweigh the haphazard methods we are obliged to pursue in general anesthesia.

There are, however, a number of conditions in which general anesthesia is necessary-which anesthetic shall we employ? In the discussion in London it was developed that chloroform had long been, and was still, in use in work of this nature. Dr. Silk believes that the routine use of chloroform is founded on the assumption that it is safest in children, and from which its use has extended to adults. Lennox Browne prefers the local anesthetic effects of cocain in intra-nasal operations, but gives a general anesthetic if it is indicated or desired.

He with Stoker, Silk, Dundas, Grant, and a number of prominent English laryngologists prefer the ase of nitrous oxid in operations requiring but a few seconds. In those requiring a longer time nitrous oxid is given to produce insensibility and to avoid the discomfort of inhaling ether-the latter is then continued as the anesthetic.

In hospital work the plan of the London surgeons is ideal, but in private practice there are many difficulties in the use of nitrous oxid, and especially in operations at the bedside.

Chloroform can not be recommended for reasons which will be more fully discussed under naso-pharyngeal operations. Bromid of ethyl is too transient in its effect for these operations.

Ether then remains alone the safest and most reliable anesthetic.

The obstacles to the management of the patient which general anesthesia presents are in a measure met by care in position. The half-reclining or sitting position is not to be recommended. The one thing that would seem to justify such position in an intra-nasal operation would be that it is the most favorable in which to employ artificial light. Against this, however, is the utter uselessness of any light after the first incision, when the parts are covered with and obscured by blood. Indeed, in these operations one is guided almost entirely by the knowledge he has gained of the parts prior to the operation.

A very cogent argument against this position is its danger-one not to be lightly set aside, especially in the use of chloroform.

The Trendelenburg position, or one with similar objects in view, is without doubt the most satisfactory for the following reasons: 1, with proper electric appliances as good illumination may be obtained in this as in any other position. At our clinic at the Episcopal Hospital an ordinary incandescent light with a reflecting surface back of the globe is placed in the hands of an assistant and the light is thrown upon the field of operation at any desired angle. The various forms of electric head-lights may be employed with equal, perhaps, greater advantage; 2 , this position causes the blood to gravitate to the post-nasal and pharyngeal spaces, whence it may be repeatedly removed by a sponge in the hands of an assistant, or, if at any time the blood flows too freely the head may be turned to one side and the blood allowed to escape from the opposite nostril or the angle of the mouth. This gravitation of the blood posteriorly not only lessens the danger of aspiration but it in a large measure frees the anterior portion of the nares, thus enabling the operator to have a clearer view of the field of operation.
General anesthesia in intra-nasal operations is but a sorry substitute for local anesthesia, and should be employed only when the necessities of the case urgently demand it.

We are obliged, at times, to meet the wishes and desires of our patients; some of whom prefer general anesthesia because the operation can be done without their consciousness. At the same time it seems to me a folly to undertake such operations merely to please the patient unless we are reasonably sure we understand beforehand every necessity of the case.

The larger number of intra-nasal operations being best undertaken under local anesthesia, we must now decide which of the few local anesthetics will serve our purposes best, and the manner of its use.

Cocain has held undisputed sway as a local anesthetic for about fifteen years and, in the main, leaves little to be desired. Those who have used it freely, however, have now and then observed effects which have given rise to anxiety, and a few fatal cases have been reported from its use.

It has always been a matter of doubt to me in these cases of cocain poisoning as to the part played by the shock of the operation. A nervous woman cuts her finger and faints at the sight of blood; this same woman should certainly be expected to faint during an operation on her septum. I believe that some of the cases which have been regarded as cocain poisoning are of this nature.

A very few can not be explained in this manner and we are forced to admit that in some subjects an idiosyncrasy exists to the drug.

More recently, eucain has been extolled as the equal of cocain in anesthetic power and, it is asserted, possesses no such tendency as the latter drug to produce intoxication.

Our experience with this new anesthetic is necessarily too limited to assert positively as to the latter claim. There is no doubt in the writer's mind as to the validity of the former.

In an article published in the Philadelphia Polyclinic, Jan. 23, 1897, the author gave an experience of six months use of this drug in the clinies of the Episcopal and Polyclinic Hospitals. It was found to have equal anesthetic power with cocain, both as to intensity and duration of anesthesia in the larger number of cases.

Its power to reduce engorged turbinates was also equal to that of cocain. In the pharynx, while it possesses equal anesthetic power to cocain, it lacks those unpleasant suffocative, choking sensations which the latter drug at times induces. It is, therefore, much pleasanter in its effects in this locality. In no case were any symptoms approaching intoxication induced.

These results have been confirmed by a riper experience. Eucain is now used at my clinics in all operations in the nares, naso-pharynx and pharynx, except in those few instances in which cocain seems to have a better effect.

Its use in the larynx has been abandoned because cocain has equal anesthetic power and is slightly less irritating and hence less apt to produce annoying and troublesome spasms.

The pleasantest manner of obtaining anesthesia in the nasal chambers is as follows: First spray both chambers with a 2 per cent. solution of whichever anesthetic we elect, to obtain tolerance for the harsher 
methods to follow. After waiting for one minute sat. urate a small pledget of cotton with a 4 to 10 per cent. solution of the drug and allow it to lie at the site of operation for from five to eight minutes.

Antipyrin in 5 per cent. solution has been found to intensify and prolong the anesthetic effect of cocain or eucain; therefore, if the projected operation promises to be a tedious one after the removal of the tampon of cotton, a spray of antipyrin ( 5 per cent.) may be passed through the nasal chambers.

Ántipvrin also has a decided hemostatic effect and in operations upon the septum or the posterior turbinates, parts that are quite vascular, this drug will prove to be an agent of decided advantage. It is, however, much too irritating for use in the nasal chamber, unless preceded by the free use of eucain or cocain.

With these two reliable local anesthetics the rhinologist is well provided, and intra-nasal surgery is robbed of much of its terrors.

Naso-pharynx.-The most frequent operative procedure the surgeon is called upon to undertake in the naso-pharynx is that for the removal of adenoid vegetations from the vault.

There is great difference of opinion existing among surgeons regarding anesthesia in these cases.

Some viewing the operation as trivial, eschew the use of anesthesia in any form; others advise a local, and still others always employ a general anesthetic.

Probably much of this confusion and difference of opinion arises from an improper selection or arrangement of the class of cases.

In deciding upon the use of an anesthetic we should be influenced by the particular form of operation we are to employ and also by the age of the patient.

In adults and children of riper years who may be properly controlled these growths are best removed by means of the postnasal forceps.

These instruments should never be employed under general anesthesia, save by those thoroughly familiar with work of this nature and then guided by a finger insinuated back of the soft palate; this latter precaution is only possible to those having small digits, and hence the application of this method is limited.

The use of the forceps without the aid of the mirror in a space hidden from view has always seemed an unsurgicial proceeding and especially since we have the means at our command to bring the parts thoroughly under the eye and to follow every step of the operation.

In those cases, therefore, of hypertrophy of the pharyngeal tonsil in adults or older children, in which it seems preferable to employ the postnasal forceps, the operation should be performed under the local anesthetic influence of eucain or cocain and by the aid of the rhinoscopic mirror. There are many adults and older children either too nervous or with pharynges ill adapted for rhinoscpic work, these then will come under the same rules as those to be presently adverted to in the case of children.

By far the larger number of cases of adenoids occur in young children. The most popular methods employed for their removal are the curette, the fingernail or both combined.

In selecting an anesthetic, or in determining whether or not to use one, the surgeon is inflenced by the estimate he attaches to the operation. In the hurry of life and in oft-repeated successes he is apt to regard this as a trivial operation and one unworthy of much time consumed in the performance. Considering the proximity of important structures and the possibility of injury to the same, does this seem good judgment?

A general surgeon would deem it the height of imprudence to operate on a case and, especially if an anesthetic were considered necessary, dismiss it with little or no directions. He would rather have the patient remain in bed at least for a day to avoid any possible complication by means of chilling the surface, a contingency very possible to a child going into the open air a short time after an operation.

Adenoid operations are of daily occurrence in our clinics with and without the use of anesthesia and patients are dismissed with little instructions. It may be said that few accidents happen as a result of such methods. It is true we see few complications, but can we be certain they do not occur among a class of patients notoriously difficult to keep in view? Have we not seen acute inflammatory troubles of the middle ear follow this operation? Is it not possible that the apparent return of the trouble within a short period of time may be due to inflammatory conditions induced by exposure after the operation?

It has always seemed that the best interests of our little patients would be served were we to regard these operations of sufficient importance to demand at least one day's stay in bed.

These views have been borne out by my experience. At the Episcopal Hospital those cases treated in this manner have been free from complications of any sort and the results have been most satisfactory.

The operation of curettement of the pharyngeal vault is a painful one; slightly less so by the use of encain or cocain. It is therefore scarcely a humane procedure without an anesthetic in young children. To forcibly hold a nervous, irritable child and subject it even for a few seconds to severe suffering is a great tax to the nervous energies of that child and might lead to serious consequences.

The pain together with the gagging produces such contraction of the phayngeal muscles as to place the parts in the most unfavorable position for good and thorough work.

General anesthesia should be employed in the removal of adenoids for the following reasons: 1 . The operation can be performed in a more efficient and satisfactory manner. 2 . It is attended with less danger of depressing effects to the nervous system of the child. 3. It serves as an extra inducement to treat these as surgical cases requiring care in the after-treatment and enables the surgeon to keep the patient in bed at least one day and thereby conduces to a successful result.

The operation can be done so quickly that the anesthetic which seems eminently suitable for these cases is nitrous oxid gas, the effects of which are transient.

In the London Throat and other hospitals in Great Britain this is the anesthetic employed, and in the discussion in London, already adverted to, the consensus of opinion was in favor of the use of this gas.

Dr. Silk ${ }^{2}$ prefers it because it is pleasant, safe and can be administered in every position of the patient. No previous preparation is necessary and the recovery from it is rapid and perfect and the after-effects nil. Lennox Browne $\theta^{3}$ uses no uncertain language in his advocacy of nitrous oxid gas in these operations. $\mathrm{He}$ 2 Silk: Journal of Laryngology, London, 1889, 1ii, p. 177-181; 238-242. 3 Browne: Med. Press and Circular, London, 1893, n. s., lvi, p. 136.
. 
says: "The rapidity with which the patient can be anesthetized by gas, the almost constant absence of any unpleasant after-effects, the exceedingly short interval required for complete recovery of consciousness in addition to the incalculably yreater safety on general grounds of its administration over any other agents, not only constitnte strong arguments for its employment where the time required for the comple. tion of the operation does not exceed from forty-five to fifty seconds, but actually renclers the use of other agents culpably unjustifiable."

Nitrous oxid has had more or less extensive trial in this country and is well thought of by those familiar with its use. In hospital practice, and especially if care in the after-treatment follows its use, nitrous oxid leaves little to be desired as an efficient and safe anesthetic in the removal of adenoid growth in young children. In private practice, however, the cumbersome nature of the apparatus necessary for the production of this gas renders its use awkward, save in office work and here its use can not be recommended for reasons already stated.

Impressed as the writer is with the advantages of treating these as any other surgical cases, where it is possible to do so, we must needs search for an anesthetic safe and reliable which may be employed at the bedside.

Chloroform has long been use and especially by the English surgeons.

The chief reasons for its use have been that it is pleasant in its effects, was supposed to be harmless in children, quick in its action and free from unpleasant after-effects. Bosworth in the recent edition of his work favors this anesthetic.

When it is considered that no less than nine deaths occurred in England in one year from the use of chloroform, it should cause the surgeon to hesitate before employing it.

Dr. Silk, the anesthetist for the Great Northern Central Hospital clearly and forcibly states the advantages and disadvantages of chloroform in these operations. I quote from his paper: "I believe that the routine use of chloroform is founded upon the assumption that it is safest for children, a general proposition with which $I$ in no way agree, and from which I fancy its use has extended to adults. Other advantages claimed for it are:

1. That it is simple to administer, and the resulting narcosis is easily maintained. It does not come within the scope of this communication to point out that these advantages are much exaggerated, but even if this were not the case I hardly think that the convenience of the operator should be placed above the safety of the patient.

2. That the bleeding and turgesence of the mucous membrane is less than when ether is used; but even if this were true the difference, as I have already pointed out is so slight that this advantage almost sinks into insignificance.

3. That the primary narcosis is more profound This should I think be classed rather as a disadvantage, inasmuch as the zone maniable, to which $I$ have already referred, is in respect to chloroform very narrow, and therefore even if no respiratory embarrassment prevailed, its use is always attended with a certain amount of risk; much more is this the case when the comparatively pure vapor which accumulates at the back of the throat may be inhaled at any time and is quite beyond our control.
The objections if not numerous are weighty:

1. I can fully endorse the view that chloroform administrations are always a source of anxiety to the anesthetist and in some instances of narrow escapes to the patients, and this is much more the case in naso-pharyngeal operations. On account of the accumulation of the blood at the back of the throat, and consequent interference with the respiratory functions, the heavy chloroform vapor speedily collects above the larynx, in a nearly pure or highly concentrated state. Hence the attacks of syncope and apnea which so commonly occur without the slightest warning, when chloroform is being administered. Its slight diffusibility, too, tends to permit of its accumulation in the lungs, and is accentuated by the res. piratory failure. Hence the attacks of syncope and apnea occurring as they frequently do, some time after the administration has been withdrawn.

2. The after-effects of chloroform are often very prolonged. Such immediate effects as nausea, the loss of blood swallowed, are probably no better and no worse than those attendant upon the administration of ether, but profound prostration and digestive disturbance are said to continue for days.

3. Its administration in any but the absolutely recumbent position is quite inadmissible.

Bromid of ethyl is highly thought of both by the German surgeons and a few in this country. So far as the quick action and pleasant effects are concerned, this drug seems peculiarly fitted for these operations.

Morgenthau ${ }^{4}$ of Chicago reports the results in 100 cases of adenoids in which bromid of ethyl was the anesthetic employed, and refers to Moritz Schmidt of Frankfurt-on-the-Main as having used it in 200 cases.

Morgenthau thinks that bromid of ethyl should not be used in chronic heart disease, 1.85 c.c. for children and 2.9 to 3.7 c.c. for adults is sufficient. In from forty to sixty seconds breathing becomes stertorous and for two minutes the patient does not feel pain. He operates in the upright position with the head inclined slightly forward so that the blood may flow out of the mouth.

He finds that the anesthesia, operation and recovery takes from five to seven minutes.

In the 100 cases of Morgenthau and 200 of Moritz Schmidt no deaths and but few unpleasant symptoms occurred.

Bromid of ethyl has as advantages, quickness of action, pleasantness both in the administration and the after-effects. The unfortunate result of the first introduction of this drug in Philadelphia by the late Dr. Levis, in which two patients succumbed, has deterred the profession there from its use. It is claimed, however, by those familiar with its use that the drug is nearly as safe as ether and that the early deaths were due to imperfections in the manufacture or in the substitution of bromid of ethylene a much more dangerous product.

The testimony of so many worthy and careful observers would seem to warrant that this drug which has so many advantages, should have a more extended trial.

Ether is the safest and perhaps the most widely useful anesthetic.

It is often unpleasant both in administration and after-effects but these are of slight importance in the security one feels in its use and in hospital work; fol-

Morgenthau: Ethyl Bromid, Journal of THE AMerican Medical Association, 1895, xxiv. 
lowing along the practice of the London surgeons its use may be preceded by the more pleasant nitrous oxid gas.

In the use of ether in removing adenoid growth from the vault of the pharynx, the anesthetic should be used to complete relaxation.

The writer is not in sympathy with those who use it short of this stage and has many times witnessed operations which have been delayed and complicated by the struggles and cries of the patient.

Once complete relaxation and insensibility have been obtained the anesthetic may be permanently withdrawn and the operation proceeded with, uninterrupted by any movement on the part of the patient. Given in this manner it is quickly recovered from and is much less liable to produce vomiting and other unpleasant effects.

The same method of procedure is applicable in those cases in which it is found necessary to remove both the faucial and pharyngeal tonsils. Here, however, it is best to push the ether a little more thoroughly than is the case where the simple adenoid operation is done, for the reason that after excision of the tonsils some little time is consumed in allowing blood to escape from the mouth before curetting the vault.

Incidentally the position of the patient during these operations is of importance. The most satisfactory results are obtained in operations in the naso-pharynx in that position which places the posterior wall of the pharynx and naso-pharynx in the most dependent position. This is accomplished by the Trendelenburg position or a modification of the same. By this method we are not delayed by gurgling of blood in the pharynx and symptoms of asphyxia and the danger to respiration is at a minimum.

At the house of our patient the true Trendelenburg position is not always feasible. In this case all that is necessary to obtain the same result is to allow the head to hang over the edge of table or bed. The blood is sponged from the pharynx by an assistant, the patient is turned on the side and the blood permitted to escape from the nose or the angle of the mouth.

Pharyngeal operations.-Nearly all the operative measures the laryngologist is called upon to perform in the pharynx can be accomplished under local anesthesia.

Eucain is to be recommended in this locality. It causes less discomfort to the patient and it is also probably less likely to give rise to unpleasant general symptoms.

Hypertrophied tonsils, the most frequent condition calling for surgical interference in the pharynx, when uncomplicated by anenoids at the vault, may readily be excised without the use of a general anesthetic.

This operation is accomplished so quickly, with so little pain (which latter may be annulled by local anesthesia) and the work can be done so effectively that it seems an unnecessary complication to induce general anesthesia.

In nervous, irritable children in whom the shock of operation and the necessary restraint might prove serious to the nervous system of the child, transient anesthesia is to be recommended. Nitrous oxid is eminently useful in cases of this nature.

Hypertrophied tonsils, associated with adenoid vegetations at the vault should be treated in a similar manner to that spoken of in the discussion on the removal of hypertrophied pharyngeal tonsils.
Laryngeal operations.-All intra-laryngeal operations are performed under local anesthesia.

Cocain is preferable in the larynx, because its anesthetic properties are equal to eucain and it is distinctly less irritating and consequently less liable to produce unpleasant spasms. By local anesthesia the modern laryngologist is enabled to remove growths formerly deemed inoperable or only removed by splitting the thyroid cartilage, thus causing subsequent impairment of the voice.

The various major operations, such as laryngectomy, thyrotomy and tracheotomy, are accomplished under general anesthesia. Recently, however, a number of tracheotomies have been done with cocain injected along the line of operation as the only anesthetic employed.

This method may occasionally be possible in adults or in children already narcotized by prolonged dyspnea, but it would be exceedingly unwise to advocate it as of general utility in children, upon whom we are most frequently called upon to do this operation.

1804 Chestnut Street.

\section{TONSIL AND ADENOID OPERATIONS UNDER ANESTHESIA BY NITROUS OXID, AND NITROUS OXID AND OXYGEN: A PRELIMINARY REPORT.}

Presented to the Section on Laryngology and Otology, at the Forty-eighth Annual Meeting of the American Medical Association. held at Philadelphia, Pa., June 1-4, 1897.

BY W. E. CASSElbERRY, M.D., AND F. MENGE, M.D. CHICAGO. ILL.

The desirability of an anesthetic that would obviate the general objections pertaining to chloroform and ether in operation on tonsils and adenoids has been the source of much research. The chief objection to chloroform lies in its danger; bromid of ethyl has scarcely attained any greater confidence; ether, while reasonably safe, is so prolonged in its effects as to be incon. venient for this purpose. Nitrous oxid alone has been employed by a few operators, notably at the Central London Thruat Hospital, but the available operating period from a single administration is almost too brief for the satisfactory removal of both the tonsils and adenoids. Experience has proven this anesthetic, however, to be safer than any other and to possess the advantage of a rapid revival of the patient; therefore following Gardner, Hunt and Hewitt of England, and Van Arsdale of New York, we have sought to modify the action of nitrous oxid by the addition of oxygen in order to render it more available and less objectionable for this operation.

As regards the safety of nitrous oxid, it is estimated by $\mathrm{H}$. C. Wood that it is given in 750,000 cases annually and we have been able to learn of but thirteen deaths fairly attributable to it.

The anesthetic effects of nitrous oxid gas were first noted by Sir Humphrey Davy, about the end of the last century, and it has since, from year to year, come into more general use.

Under nitrous oxid the respiration becomes slower and if the gas be pushed, a complete cessation of respiratory movements eventually takes place. The heart beats quietly, fully and regularly under this gas. The pulsations are somewhat slow in profound narcosis. There is, however, but slight danger from heart failure resulting from the inhalation. 